\title{
Adrenal medulla grafts enhance functional activity of the striatal dopamine system following substantia nigra lesions
}

\author{
Jill B. Becker ${ }^{1}$ and William J. Freed ${ }^{2}$ \\ 'The University of Michigan, Department of Psychology and Neuroscience Program, Ann Arbor, MI (U.S.A.) and \\ 'The National Institute of Mental Health, St. Elizabeth's Hospital, Washington, DC (U.S.A.)
}

(Accepted 5 July 1988)

Key words: Adrenal medulla graft; Parkinson's disease; Microdialysis; Dopamine release; Striatum; Cerebrospinal fluid

\begin{abstract}
Adrenal medulla grafts in the lateral ventricle reduce the behavioral manifestations of striatal dopamine depletion in an animal model of Parkinson's disease. Using microdialysis in freely moving rats, the present experiments determined that dopamine was not detectable in cerebrospinal fluid (CSF). However, adrenal medulla grafts were associated with an increase in dopamine turnover and amphetamine-stimulated striatal dopamine release was increased in animals with behaviorally effective adrenal medulla grafts. Therefore, adrenal medulla grafts increase striatal dopamine activity without an appreciable release of dopamine into the CSF. Adrenal medulla grafts also increased serum dopamine concentrations, and the increase in serum dopamine was directly correlated with the behavioral efficacy of the grafts. We suggest that dopamine, produced by adrenal medulla grafts, may gain access to the striatum via the blood supply and then leak out into the host striatum through permeable blood vessels adjacent to the graft. Through this mechanism, adrenal medulla grafts may increase functional dopaminergic activity in the striatum. These results may be important for understanding how autografts of adrenal medulla cells produce a putative alleviation of the symptoms of Parkinson's disease.
\end{abstract}

Adrenal medulla grafts adjacent to the striatum in the lateral ventricle have been found to reduce the behavioral asymmetry associated with unilateral striatal dopamine (DA) depletion in an animal model of Parkinson's disease?. Very little is known, however, about the mechanism through which adrenal medulla grafts produce their beneficial effects. When the adrenal medulla is isolated from the adrenal cortex, chromaffin cells exhibit an altered phenotype with neuronal-like processes and an increased accumulation of $\mathrm{DA}^{6,12,18}$. In addition, in the striatum adjacent to an adrenal medulla graft, there is a gradient away from the graft of decreasing DA concentrations ${ }^{3.6}$. These findings have led to the hypothesis that DA released from grafted adrenal chromaffin cells diffuses non-selectively into the cerebrospinal fluid (CSF) and the DA-depleted striatum to decrease DA receptor supersensitivity, thereby me- diating the behavioral changes observed. The present results indicate that behaviorally effective adrenal medulla grafts do not increase CSF or striatal extracellular concentrations of DA, but they do increase the striatal DA response to amphetamine (AMPH) stimulation, suggesting enhanced functional activity of the striatal DA system.

Microdialysis in the lateral ventricle of freely moving rats was used to investigate whether adrenal medulla grafts increase CSF concentrations of DA. Female Long-Evans rats received unilateral 6-hydroxydopamine (6-OHDA) lesions of the substantia nigra, were tested for AMPH-stimulated rotational behavior and underwent microdialysis in the lateral ventricle to sample CSF (see legend to Fig. 1 for additional details). A dialysis probe was inserted into the lateral ventricle via a chronically implanted guide cannula while the animal was lightly anesthetized

Correspondence: J.B. Becker, The University of Michigan, Department of Psychology, Neuroscience Laboratory Building, 1103 E. Huron, Ann Arbor, MI 48104-1687, U.S.A. 
with ether ${ }^{2}$. The animal recovered immediately and was placed in a testing cylinder $(12 \mathrm{~cm}$ in diameter). An artificial CSF solution flowed through a liquid swivel to the probe at a rate of $1 \mu \mathrm{l} / \mathrm{min}$. Samples were collected at $20 \mathrm{~min}$ intervals. The dialysis probe was removed at the end of the day and the guide cannula was resealed with a stylet.

One to three weeks after the dialysis procedure, animals received grafts of either adrenal medulla or adrenal cortex. Adrenal tissue from two female rats (2-3 months old) of the same strain (i.e. 4 adrenal medullas or an equal volume of adrenal cortex) was inserted into the ventricle via a chronically implanted guide cannula rostral to the cannula used for dialy$\mathrm{sis}^{3,7}$. Two to 3 months after the graft, animals were retested for rotational behavior and the dialysis procedure was repeated. The adrenal medulla grafts produced a $-27 \pm 15 \%$ decrease $(n=7$; mean \pm S.E.M.) in AMPH-stimulated rotational behavior. This was significantly different $\left(t_{13}=2.69 ; P=0.02\right)$ from the $+17 \pm 5 \%$ increase $(n=8)$ in rotational behavior found for animals with adrenal cortex grafts. At the end of the experiment striatal DA content was measured by high-performance liquid chromatography with electrochemical detection (HPLC-EC) and all animals had greater than $95 \%$ DA depletion.

It had been anticipated that DA would be readily detectable in the CSF following adrenal medulla grafts, but this was not the case. Even with an assay sensitivity of 1-5 pg DA, dialysate from the CSF of adrenal medulla-grafted animals did not contain detectable amounts of DA. Thus, adrenal medulla grafts in the lateral ventricle do not release measurable quantities of DA into the CSF. Baseline CSF concentrations of the DA metabolite dihydroxyphenylacetic acid (DOPAC) were, however, elevated in animals with adrenal medulla grafts. Since DOPAC in the CSF can reflect changes in DA metabolism we investigated the effect of adrenal medulla grafts on DA turnover as indicated by the CSF accumulation of acidic DA metabolites following probenecid treatment ${ }^{8}$. As is illustrated in Fig. 1, the accumulation of DA metabolites in the CSF after probenecid was significantly higher in animals with adrenal medulla grafts compared to pre-graft values $\left(F_{1.23}=\right.$ $6.031, P=0.022)$ or compared to animals with adrenal cortex grafts $\left(F_{1,13}=4.89, P=0.0456\right)$. Therefore, DA turnover was increased in animals that re-

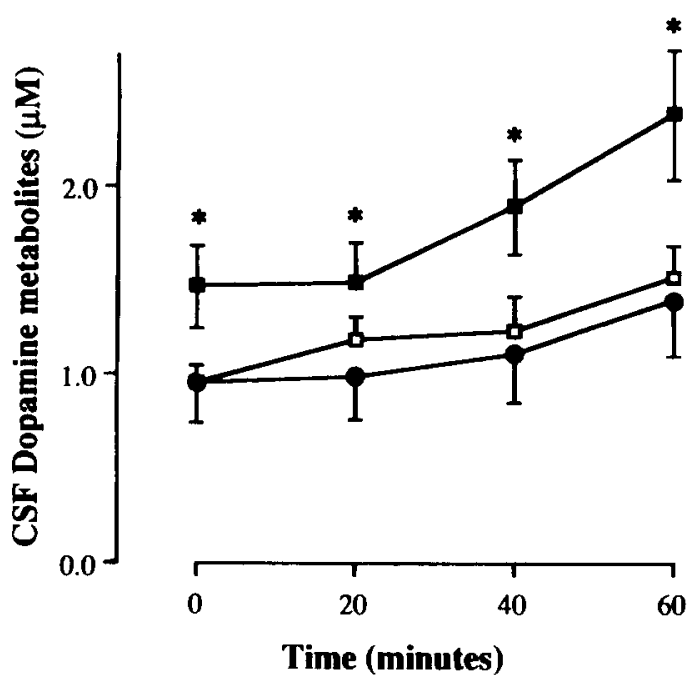

Fig. 1. Accumulation of DA metabolites in CSF following probenecid treatment. Female Long-Evans rats $(200-225 \mathrm{~g}$; Charles River) received unilateral 6-OHDA lesions of the right or left substantia nigra ( $8 \mu \mathrm{g} / 4 \mu 1$ 6-hydroxydopamine $\cdot \mathrm{HBr})$ under pentobarbital anesthesia supplemented with methoxyflurane using standard aseptic stereotaxic procedures. Three weeks later animals were screened for lesion success with 0.85 $\mathrm{mg} / \mathrm{kg}$ D-amphetamine-sulfate (AMPH). Animals that made $>50$ full rotations/h contralateral to the non-lesioned side received implants of two 18 gauge guide cannulae, both aimed at the lateral ventricle on the side of the lesion. Animals were tested for AMPH-stimulated $(3.0 \mathrm{mg} / \mathrm{kg}$ ) rotational behavior 2 weeks later in automated rotometers. The groups did not differ in their rotational behavior pre-graft (medulla group: $2290 \pm$ 274 rotations $/ 2 \mathrm{~h}$; control group: $2029 \pm 240$ rotations $/ 2$ h). Animals then underwent microdialysis and one to three weeks later received grafts of adrenal medulla or adrenal cortex tissue. The behavioral testing and microdialysis were repeated 2-3 months later. CSF concentrations of DA metabolites prior to receiving grafts (open squares) and again after grafts of either adrenal medulla (closed squares) or adrenal cortex tissue (closed circle) are illustrated. Baseline samples were obtained at time ' 0 '. Animals then received probenecid $(200 \mathrm{mg} / \mathrm{kg}$, i.p.) to block the efflux of acidic metabolites from CSF and samples were collected at $20 \mathrm{~min}$ intervals for $1 \mathrm{~h}$. Dialysate from the CSF was assayed for DA, DOPAC and homovanillic acid (HVA) before and after acid hydrolysis (to remove conjugated groups ${ }^{11}$ ) by HPLC with electrochemical detection as described previously ${ }^{2}$. Values were corrected for the rate of recovery, determined in vitro for each probe prior to implantation in the animal and are expressed as $\mu \mathrm{M}$ concentrations of total DOPAC + HVA in CSF. At no time was DA detectable in dialysate from CSF. *At all times animals with adrenal medulla grafts $(n=7)$ had significantly higher CSF concentrations of DA metabolites than animals that received adrenal cortex grafts $(n=8)$ or the same animals pre-graft $(n=17$; medulla + cortex groups combined; two animals did not complete the experiment). On a two-way analysis of variance with repeated measures over time, the main effect was significant, with no interaction $\left(F_{2.30}=3.512, P=0.0426\right)$. An elevation in the baseline concentrations of DOPAC in animals with adrenal medulla grafts $(P<0.006$, compared to pre-graft or control graft concentrations) was responsible for the difference in baseline concentrations of DA metabolites (see also ref. 2). Both DOPAC and HVA were elevated compared to the control groups following probenecid treatment. Bars indicate the S.E.M. 
ceived adrenal medulla grafts, but not in animals that received grafts of adrenal cortex (Fig. 1).

The results of microdialysis in the lateral ventricle suggested that it is not the passive diffusion of large quantities of DA from adrenal medulla grafts into the CSF and surrounding tissues that is responsible for the behavioral effectiveness of these grafts, because DA was not detectable in CSF. Nevertheless, DA turnover was elevated, so the presence of these grafts must have promoted functional DA activity in the brains of these animals. Is there increased functional DA activity in the lesioned striatum adjacent to an adrenal medulla graft? In order to address this question, extracellular concentrations of DA in the striatum were measured by microdialysis in freely moving rats with adrenal medulla or control grafts. Animals received unilateral 6-OHDA lesions, underwent behavioral testing with AMPH $(2.0 \mathrm{mg} / \mathrm{kg})$ and received grafts of either adrenal medulla or adrenal cortex tissue as described above. A dialysis probe ( $250 \mu \mathrm{m}$ outer diameter ${ }^{14}$ ) was inserted through a 20 gauge guide cannula into the striatum, $1 \mathrm{~mm}$ lateral to the ventricle, while the animal was anesthetized with methoxyflurane. The probe was secured in place with dental acrylic. Animals were placed in the testing cylinder and allowed to recover for $12-18 \mathrm{~h}$ prior to sample collection to allow injury-induced release to subside. Since grafts of adrenal medulla tissue in the lateral ventricle did not always result in a decrease in rotational behavior, animals were assigned to one of three groups based on the type of graft received and their behavior approximately two months post-graft: (1) animals with adrenal medulla grafts that showed a decrease in rotational behavior (Medulla: Decrease; $n=6$; mean change in behavior \pm S.E.M. $=-50 \pm 8 \%$; (2) animals with adrenal medulla grafts that did not show a decrease in rotational behavior (Medulla: No Change; $n=5$; mean change in behavior $=+29 \pm 6 \%)$; and (3) animals with adrenal cortex grafts (Cortex; $n=4$; mean change in behavior $=+4 \pm 12 \%$ ). When rotational behavior was compared with a one-factor ANOVA, the 3 groups of animals were significantly different $\left(F_{2.12}=24.885, P\right.$ $<0.001$ ). Subsequent pairwise comparisons indicated that the cortex group and the Medulla:No Change group did not differ from each other in rotational behavior post-graft, but both groups were significantly different from the Medulla:Decrease group $(P<0.001$; Scheffe $F$-test). The increase in AMPH-stimulated rotational behavior found in the two control groups is typical of the behavioral effect of repeated AMPH treatment (i.e. with repeated exposure to AMPH, animals exhibit a greater behavioral response to each subsequent exposure ${ }^{13}$ ). The decrease in rotational behavior in the Medulla:Decrease group, therefore, indicates that the grafts overcame both the asymmetry in striatal DA and the behavioral sensitization of the response to AMPH.

After obtaining baseline dialysis samples, animals received AMPH $(2.0 \mathrm{mg} / \mathrm{kg})$ to stimulate DA release and samples were collected for $2 \mathrm{~h}$ at $20 \mathrm{~min}$ intervals. The 3 groups did not differ in baseline concentrations of extracellular DA (values given in the legend to Fig. 2). The AMPH-induced increase in striatal DA release, however, was significantly greater for the Medulla:Decrease animals than for the Cortex group $\left(F_{1,8}=7.869, P=0.023\right)$ or the Medulla: No Change group $\left(F_{1,9}=17.638, P=0.0023\right)$ during the first hour after AMPH. The two control groups did not differ $\left(F_{1,7}=1.25, P=0.30\right)$. The small increase in striatal DA release found in the Medulla: No Change and cortex groups is thought to represent the amount of DA that the DA-depleted striatum is capable of releasing in response to AMPH. This idea is supported by a recent report ${ }^{14}$ in which it was found that even after $95 \%$ DA depletion there is significant AMPH-stimulated DA release from the striatum. The increased AMPH-stimulated striatal DA release in the Medulla: Change group, therefore, represents the contribution of the graft. To summarize, behaviorally effective adrenal medulla grafts increase AMPH-stimulated DA release in the striatum and increase CSF concentrations of DA metabolites, but do not increase basal concentrations of DA in CSF or striatal extracellular fluid.

If adrenal medulla grafts were synthesizing large quantities of DA and DA was passively diffusing from the graft into the CSF and striatum, one would expect CSF and striatal extracellular concentrations of DA to be elevated under basal conditions, but this was not the case. Adrenal medulla grafts increased DA turnover and increased the striatal DA response to $\mathrm{AMPH}$, without increasing basal DA values in CSF or striatum. How is this increase in striatal DA activity occurring? There are at least two possibilities. One is that adrenal medulla grafts induce re- 


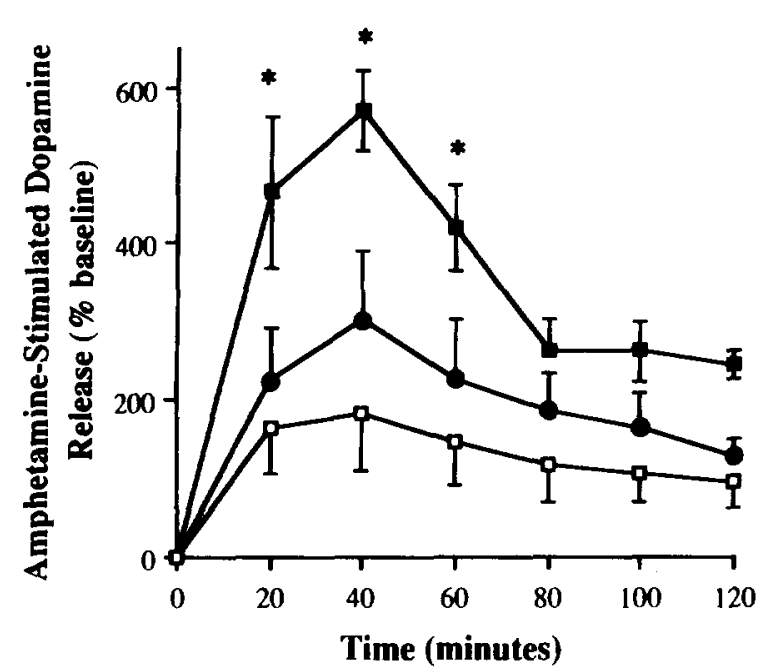

Fig. 2. AMPH-stimulated increase in extracellular DA concentrations in striatum as determined by microdialysis in freely moving rats. Two weeks after unilateral 6-OHDA lesions animals were treated daily for 10 days with AMPH $(3.0 \mathrm{mg} / \mathrm{kg})$ in their home cages to decrease sensitization of the behavioral response to $\mathrm{AMPH}^{13}$. Four weeks after the lesion animals were screened for lesion success with $1.25 \mathrm{mg} / \mathrm{kg}$ AMPH. Only animals making $>50$ rotations $/ \mathrm{h}$ on both of two trials were used in the experiment. Animals were then tested twice with $2.0 \mathrm{mg} / \mathrm{kg}$ AMPH. One week later, animals received grafts of adrenal medulla or adrenal cortex tissue. Behavioral testing was repeated 6 and 8 weeks after the graft and animals underwent intrastriatal microdialysis 1 week after the second behavioral test. Postmortem analysis of striatal DA concentrations confirmed that groups did not differ in the extent of the lesion. Microdialysis samples were collected at 20 min intervals and DA content was assayed by $\mathrm{HPLC}-\mathrm{EC}^{2}$. Baseline samples were collected at time ' 0 '. Animals then received $2.0 \mathrm{mg} / \mathrm{kg}$ AMPH and samples were collected for $2 \mathrm{~h}$. The response to AMPH is expressed as a percent of baseline DA concentrations for each of the 3 groups: Medulla:Decrease (closed squares), Medulla:No Change (open squares), and Cortex (closed circles). There were no significant between-group differences in baseline DA concentrations (mean \pm S.E.M.; Medulla: Decrease, $2.4 \pm 0.9 \mathrm{nM}$; Medulla: No Change, $3.7 \pm 0.6 \mathrm{nM}$; Cortex, $5.0 \pm 1.4 \mathrm{nM}$ ). ${ }^{*}$ The percent increase in AMPH-stimulated DA release was significantly greater $(P<0.02)$ for the Medulla:Decrease group than for the two comparison groups at these times. Bars indicate the S.E.M.

growth of striatal dopaminergic fibers as has been reported by Bohn and her colleagues for the MPTP lesioned mouse 4 . This explanation would suggest that regenerating DA fibers mediate the increase in AMPH-stimulated DA release found in animals with adrenal medulla grafts. If this is happening in the present model system, then one might expect to see enhanced catecholamine fluorescence in the striatum adjacent to adrenal medulla grafts, but this phenom- enon has not been observed in the 6-OHDA-lesioned rat $^{3,6,7}$. It is nevertheless possible that DA release and turnover are greatly increased in a relatively small number of regenerated DA neurites. Therefore, while we cannot rule out the regrowth of DA neurites, other mechanisms should be considered.

One possible mechanism is that DA secreted by the grafted adrenal medulla cells gains access to the striatum via the local circulatory system. Survival of adrenal medulla grafts depends on the development of a blood supply by anastomosis between blood vessels of the grafted tissue and blood vessels of the host ${ }^{15}$. The anastomosis between grafted peripheral tissue and striatal blood vessels results in a loss of the blood-brain barrier at or near the junction and an apparent increase in the permeability of the associated host bood vessels to blood-borne proteins ${ }^{16}$. Therefore, the integrity of the blood-brain barrier is compromised not only within the graft but also in the host brain adjacent to the graft. This loss of the bloodbrain barrier could allow DA released from adrenal chromaffin cells to enter the striatum through the local blood supply.

In order to test this hypothesis serum DA concentrations were measured in peripheral blood of animals with adrenal medulla grafts. In the experiments described above, adrenal medulla grafts were shown to increase AMPH-stimulated striatal DA and decrease AMPH-stimulated rotational behavior. Rotational behavior was induced by AMPH so that the changes in AMPH-stimulated striatal DA release could be shown to have an impact on an animal's behavior. However, most of the research investigating the behavioral effects of adrenal medulla grafts has used apomorphine to induce rotational behavior (e.g. refs. 6, 7). Therefore, in order to have results that would be applicable to the larger body of literature on adrenal medulla grafts, rotational behavior was induced with apomorphine in the following experiment. Male Sprague-Dawley rats (150-200 g) received unilateral 6-OHDA lesions of the substantia nigra under ketamine plus xylazine anesthesia and either a bilateral adrenalectomy or a sham operation. Adrenalectomized rats were maintained on $1 \%$ saline as the sole source of fluid. Beginning 6 weeks after the lesion, animals were tested for rotational behavior following $0.1 \mathrm{mg} / \mathrm{kg}$ apomorphine (s.c.) for 6 test sessions of $60 \mathrm{~min}$ each. Four months after the le- 


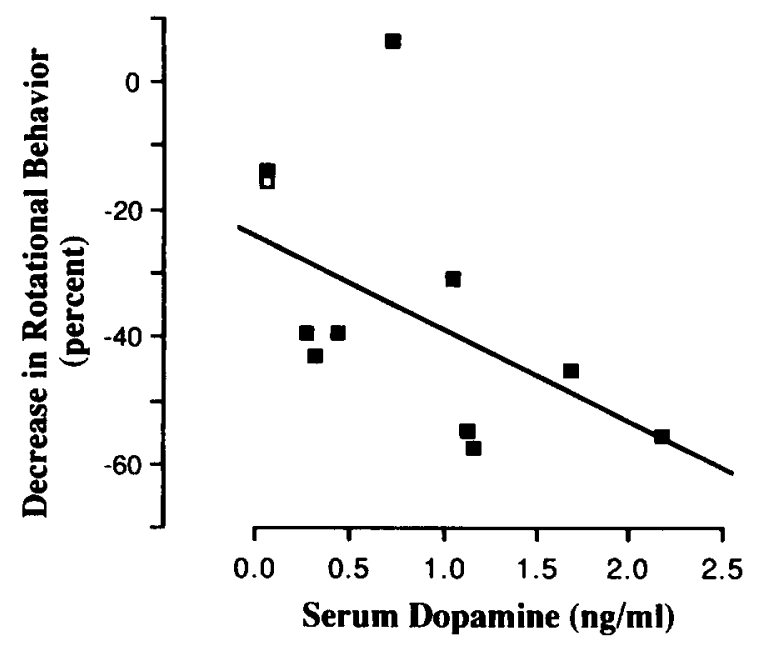

Fig. 3. Correlation between serum DA concentrations and adrenal medulla graft-induced change in rotational behavior. Blood samples $(2 \mathrm{ml})$ were collected with heparinized capillary tubes from the infraorbital sinus under ether anesthesia. DA was stabilized by the addition of $7.5 \mathrm{mg}$ EDTA to each tube. Samples were centrifuged and $50 \mu \mathrm{l}$ of $0.05 \mathrm{M} \mathrm{HClO}_{4}$ containing $500 \mathrm{pg}$ dihydroxybenzylamine (as an internal standard) were added to a measured volume of serum. Catecholamines in serum were purified by alumina extraction and concentrations of DA in serum were determined by HPLC-EC as described previously ${ }^{2}$. Serum concentrations of DA were directly correlated (rho $=-0.72, P<0.05$ ) with the decrease in apomorphine-induced rotational behavior following adrenal medulla grafts. DA concentrations are expressed as $\mathrm{ng} / \mathrm{ml}$ serum. The change in rotational behavior is expressed as a percent of pregraft rotational behavior. Each square represents the data from one individual animal $(n=11)$. Data were analyzed using Spearman's rank correlation coefficient test. Probabilities reported are 2-tailed.

sions, animals received intraventricular adrenal medulla grafts as previously described ${ }^{6}$. Six weeks after transplantation animals received 4 additional tests of apomorphine-induced rotation. Blood samples were drawn 10 weeks after transplantation (see legend to Fig. 3 for details).

Adrenal medulla grafts produced decreased apomorphine-induced rotational behavior $(n=11$; mean change in behavior \pm S.E.M. $=-36.8 \pm 6.5 \%)$ and elevated serum DA concentrations $(0.763 \pm 0.217$ $\mathrm{ng} / \mathrm{ml}$ ) compared to animals without adrenal medulla grafts $(n=4 ; 0.169 \pm 0.114 \mathrm{ng} / \mathrm{ml})$. More importantly, as illustrated in Fig. 3, serum concentrations of DA were directly correlated (rho $=-0.72, P<0.05$ ) with the magnitude of the change in rotational behavior. In other words, animals with the highest serum DA concentrations showed the greatest decrease in rotational behavior.

The behavioral effect of these grafts and the increase in serum DA concentrations were not dependent on the presence of the host animal's adrenal gland. Adrenalectomized animals with adrenal medulla grafts also showed both a decrease in rotational behavior $(n=11 ;-51.2 \pm 12.9 \%)$ and an elevation in serum DA concentrations $(0.829 \pm 0.289 \mathrm{ng} / \mathrm{ml})$. In contrast, serum DA was not detectable in adrenalectomized animals without adrenal medulla grafts $(n$ $=6$ ). Interestingly, adrenalectomized rats tended to show greater variability in the behavioral effect of adrenal medulla grafts (range $=+58.2 \%$ to $-90.8 \%$ change in rotations compared with $+4.6 \%$ to $-59.1 \%$ change for the sham-operated rats) so the correlation between serum DA and the change in rotational behavior was not statistically significant in this small number of animals. When data from all of the adrenal medulla grafted animals is pooled, however, the correlation between serum DA concentrations and percent decrease in rotational behavior is maintained (rho $=-0.444 ; P<0.05$ ). Fluorescence histochemistry ${ }^{5}$ and tyrosine hydroxylase immunocytochemistry ${ }^{17}$ verified that substantial numbers of surviving adrenal chromaffin cells (less than 30 to several thousand) were present 3-5 months after transplantation in all but one of the 10 animals examined. These data strongly suggest that DA produced by adrenal medulla grafts enters the circulatory system. Furthermore, because the behavioral efficacy of these grafts is directed correlated with serum DA concentrations it would appear that DA from adrenal medulla grafts secreted into the circulation produces a change in the animal's behavior.

In conclusion, adrenal medulla grafts increase DA turnover, AMPH-stimulated striatal DA release, and DA concentrations in blood without producing an increase in CSF or striatal extracellular concentrations of DA. Moreover, the concentrations of DA in blood and the restoration of the striatal DA response to AMPH are related to the behavioral efficacy of the grafts. Interest in the mechanism through which adrenal medulla grafts alleviate the symptoms of DA depletion has increased with the reports of clinical trials using autografts of adrenal medulla tissue for the treatment of patients with Parkinson's disease $^{1.9 .10}$. In only one of these studies, however, has 
significant long term improvement in the symptoms of Parkinson's disease been reported ${ }^{10}$. Delineation of the mechanism(s) involved in adrenal medulla graft efficacy may help to increase the success of clinical procedures. While other beneficial effects of these grafts cannot be ruled out at this time, the results presented here suggest that the production of DA by adrenal medulla grafts plays an important role in their behavioral efficacy.

1 Backlund, E.-O., Granberg, P.-O., Hamberger, B., Knutsson, E., Martenson, A., Sedvall, G. and Olson, L., Transplantation of adrenal medullary tissue to striatum in parkinsonism. First clinical trials, J. Neurosurg., 62 (1985) $169-173$.

2 Becker, J.B., Adams, F. and Robinson, T.E., Intraventricular microdialysis: a new method for determining the concentrations of monoamine metabolites in the CSF, J. Neurosci. Methods, 24 (1988) 259-269.

3 Becker, J.B. and Freed, W.J., Neurochemical correlates of behavioral changes following intraventricular adrenal medulla grafts: in vivo microdialysis in freely moving rats, Prog. Brain Res., in press.

4 Bohn, M.C., Cupit, L., Marciano, F. and Gash, D.M., Adrenal medulla grafts enhance recovery of striatal dopaminergic fibers, Science, 237 (1987) 913-916.

5 De la Torre, J.C., An improved approach to histofluorescence using the SPG method for monoamines J. Neurosci. Methods., 3 (1980) 1-5.

6 Freed, W.J., Functional brain tissue transplantation: reversal of lesion-induced rotation by intraventricular substantia nigra and adrenal medulla grafts with a note on intracranial retinal grafts, Biol. Psychiatry, 18 (1983) 1205-1267.

7 Freed, W.J., Morihisa, J.M., Spoor, H.E., Hoffer, B.J., Olson, L., Seiger, A. and Wyatt, R.J., Transplanted adrenal chromaffin cells in rat brain to reduce lesion-induced rotational behavior. Nature (Lond.), 292 (1981) 351-352.

8 Hutson, P.H., Sarna, G.S., Kantamaneni, B.D. and Curzon, G., Concurrent determination of brain dopamine and 5 -hydroxytryptamine turnovers in individual freely moving rats using repeated sampling of cerebrospinal fluid, $\mathrm{J}$. $\mathrm{Neu}$ rochem., 43 (1984) 151-159.

9 Lindvall, O., Backlund, E.-O., Farde, L., Sedvall, G., Freedman, R., Hoffer, B., Nobin, A., Seiger, A. and Olson, L., Transplantation in Parkinson's Disease: two cases of adrenal medullary grafts to the putamen, Ann. Neurol.,
We would like to thank Dr. T.E. Robinson for designing the microdialysis probe used in these studies and for valuable discussions. Our thanks also to Drs. E.S. Valenstein and T.E. Robinson for their comments on the manuscript. This research was supported by grants to Dr. Becker from the NIH (NS22157) and the American Parkinson's Disease Association. Dr. Becker is supported by a Research Career Development Award from the NIH (NS01056).

22 (1987) 457-468.

10 Madrazo, I., Drucker-Colin, R., Diaz, V., Martinez-Mata, J., Torres, C. and Becerril, J.J., Open microsurgical autograft of adrenal medulla to the right caudate nucleus in two patients with intractable Parkinson's Disease, N. Engl. J. Med., 316 (1987) 831-834.

11 Nagel, M. and Schumann, H.-J., A sensitive method for determination of conjugated catecholamines in blood plasma, J. Clin. Chem. Clin. Biochem., 18 (1980) 431-432.

12 Olson, L., Fluorescence histochemical evidence for axonal growth and secretion from transplanted adrenal medullary tissue, Histochemie, 22 (1970) 1-7.

13 Robinson, T.E. and Becker, J.B., Enduring changes in brain and behavior produced by chronic amphetamine administration: a review and evaluation of animal models of amphetamine psychosis, Brain Res. Rev., 11 (1986) 157-198.

14 Robinson, T.E. and Whishaw, I.Q., Normalization of extracellular dopamine in striatum following recovery from a partial unilateral 6-OHDA lesion of the substantia nigra: a microdialysis study in freely moving rats, Brain Research, 450 (1988) 209-224.

15 Rosenstein, J.M., Adrenal medulla grafts produce bloodbrain barrier dysfunction, Brain Research, 414 (1987) 192-196.

16 Rosenstein, J.M. and Brightman, M.W., Alterations of the blood-brain barrier after transplantation of autonomic ganglia into the mammalian central nervous system, J. Comp. Neurol, 250 (1986) 339-351.

17 Sternberger, L.A., Immunocytochemistry, Wiley, New York, 1979.

18 Unsicker, K., Krisch, B., Otten, U. and Thoen, H., Nerve growth factor-induced fiber outgrowth from isolated rat adrenal chromaffin cells: impairment by glucocorticoids, Proc. Natl. Acad. Sci. U.S. A., 75 (1978) 3498-3502. 